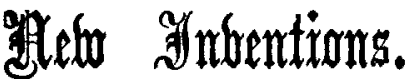

\section{THE RED CROSS AMBULANCE.}

Mr. JoHN Carter, of 6A, New Cavendish-street, forwards as for inspection a sample of his new Red Cross Ambulance, which has only recently been brought into the market. The steel springs, so that vibration is reduced to a minimum. The bicycle wheels are constructed in the most approved fashion and, indeed, the mechanism of the apparatus would seem to leave nothing to be desired. One advantage of the ambulance is that the material of which it is composed admits of the removal of infectious cases, since its construction, being of light iron, serves to lessen risk of infection. Another

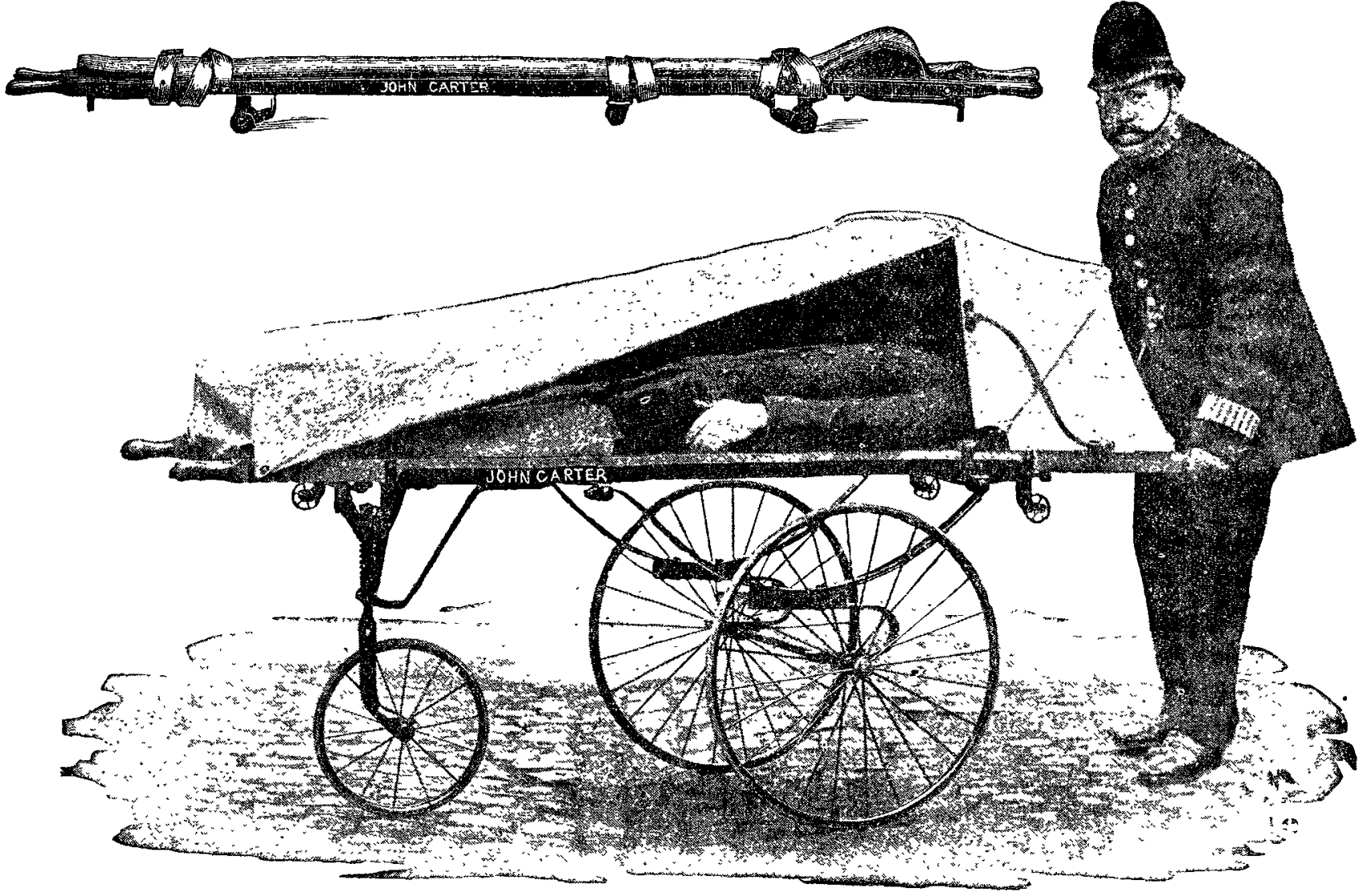

acesmpanying illustration will satisfactorily set forth in the important feature is the arrangement of the hood, which is rough the parts and properties of this contrivance. A few jointed in the same manner as are the hoods used in Bath particulars, however, may well be supplemented, by way of chairs. We can recommend the device to the notice of all verbal description. The carriage is mounted on half-elliptic those who are interested in ambulance work.

\section{THE STERILISATION OF SOLUTIONS FOR} OPHTHALMIC USE.

THE eye-drop flasks which were recently introduced by Dr. Stroschein of Würzburg possess certain obvious advantages over those in common use. The fact may be mentioned that these bottles were shown at the January meeting of the Oph. thalmological Society, but the invention seems to me to deserve the notice of the profession at large. The flask is constructed of thin glass, so as to bear heat without breaking. A pipette ats into the flask and differs from those commonly employed in that it has two bulbs instead of one. The pipette, which is of course traversed by a central channel, is surmounted by an india-rubber nipple. In order to sterilise the contained solution the nipple is removed and the pipette is reversed and inserted into the flask. The whole apparatus is then boiled over a small flame-e.g., that of a spirit lamp-for three minutes. During this process the flask may be held by a wooden clip or it may be supported by wire gauze on a tripod. As soon as boiling point is reached the steam rises from the liquid and passes through the pipette, which is thus effectually sterilised. Thirty seconds after the removal of the bottle from the flame the pipette is inserted in its original position. Should time, however, be a matter of consequence, the bulb may be more speedily cooled by allowing cold water to run over its surface. Finally, the india-rubber teat is replaced and the bottle is ready for use. If the bottle is in constant ase the boiling process must, of course, be repeated at inter- vals of a few days. Repeated sterilisations must of necessity concentrate the solutions. Dr. Stroschein meets this difficulty by adding from eight to ten drops of distilled water to every twenty cubic centimetres of the liquid before it is boiled. Two further points may be mentioned: first, that the name of the contained solution is indelibly inscribed upon the face of each flask; and, secondly, that the bottles vary in colour according to the solution they are intended to contain-thus the bottle for atropine is black, that for cocaine is white, for physostigmine it is red, and for homatropine it is blue. That the process described secures absolute sterilisation of the bottle, contents and pipette is attested by a series of more than one hundred bacteriological experiments conducted by Dr. Stroschein. The flasks are made by Otto Wiegand of Würzburg. They can be obtained in this country from Messrs. Schollar and Simsky, 64, Praed-street, W. A complete set, consisting of four bottles and a tripod, costs $9 s .6 d$. ; a single Hlask is sold at $2 s$. Welbeck-street, W. Surgeon to the Hanwell Ophthalmic School.

Vaccination Awards.-MI:. Percy Howard Day, medical officer and public vaccinator for the Stalmine district of the Garstang union, has for the third time in succession been awarded the Government grant for efficient vaccination. Mr. J. 'T. Roger Miller has received an award from the Local Government Board for public vaccination in the Leavening district of the Malton union. 